\title{
ZAP70 Expression Within del6q21, del11q13 and del17p13 Cytogenetic Subgroups of Iranian Patients with Chronic Lymphocytic Leukemia
}

\author{
Hamideh Jafari Ghahfarokhi ${ }^{1}$; Saeede Ashoori ${ }^{\text {; }}$; Mohamad Taghi Akbari ${ }^{2}$; Masoud \\ Lotfizadeh $^{3}$; Ali Karimi ${ }^{4}$; Hossein Teimori ${ }^{1, *}$ \\ ${ }^{1}$ Cellular and Molecular Research Center, Shahrekord University of Medical Sciences, Shahrekord, IR Iran \\ ${ }^{2}$ Department of Medical Genetics, Faculty of Medical Sciences, Tarbiat Modares University, Tehran, IR Iran \\ ${ }_{3}^{3}$ Department of Public Health, Social Health Determinants Research Center, School of Health, Shahrekord University of Medical Sciences, Shahrekord, IR Iran \\ ${ }^{4}$ Medical Plant Research Center, Shahrekord University of Medical Sciences, Shahrekord, IR Iran \\ *Corresponding Author: Hossein Teimori, Cellular and Molecular Research Center, Shahrekord University of Medical Sciences, Shahrekord, IR Iran. Tel: +98-3813346692, Fax: +98- \\ 3813330709, E-mail: hosseintimm@yahoo.com
}

Received: August 4, 2014; Revised: September 10, 2014; Accepted: September 28, 2014

\begin{abstract}
Background: B-cell chronic lymphocytic leukemia (B-CLL) is the most common form of leukemia in adults. Some reports showed that expression of ZAP70 gene and chromosomal abnormality are two prognostic factors in management of B-CLL

Objectives: In this study, we determined ZAP70 mRNA expression level in the del17p13, del6q21 and del11q13 subgroups of Iranian B-CLL patients to investigate prognostic value of ZAP70 expression.

Patients and Methods: In this cross-sectional study, fluorescence in situ hybridization analysis was carried out on 66 Iranian B-CLL patients. Zap70 mRNA expression was evaluated by using Real Time RT-PCR.

Results: Molecular analysis showed that ZAP70 expression increased 2.46 fold in the del11q13 subgroup, 2.87 fold in the del17p13 and 1.87 fold in the del6q21, compared to the 15 patients in the control group. Comparison of standard deviation and mean of the ZAP70 expression profile within the subgroups showed more variability among the cases of the del11q13 and del17p13 versus tight clustering for the del6q21. Therefore, there is a relation between del6q21 aberrations; which has good prognosis with normal levels of ZAP70 expression.

Conclusions: The results of ANOVA test showed that ZAP70 expression gene was significantly increased in del17p13 and del11q13 subgroups compared to control group. Thus, ZAP70 may play an important role in the prognosis of B-CLL patients.
\end{abstract}

Keywords:Chronic Lymphocytic Leukemia; ZAP70 Expression; Chromosomal Abnormality

\section{Background}

Leukemia is a kind of cancer that begins in cells that form new blood cells. These cells are detected in the soft, inner part of the bones called the bone marrow. Any blood-forming cell can transform into a leukemic cell. Once transformed, the cell can proliferate and lead to form new cancer cells. These cells can leak into the blood stream and expand to other organs. Patients with chronic lymphocytic leukemia (CLL) were first described in the early nineteenth century (1-3). The natural history of the disease progress is very variable, ranging from an indolent disorder with minimal progress over many years, to a more offensive disease with poor reaction to treatment (4). Although the leading causes of this disease are unknown, there is some evidence indicating that genetic factors play an important role in the occurrence of this disease. Clonal chromosomal abnormalities have been observed in leukemic cells of the patients with CLL, of which del 13q14-23.1 is the most common, followed in frequency by trisomy 12, del 11q22.3-q23.1, del 6q21-q23, del 17p13.1, and 14q abnormalities (5). The onset of disease average age is approximately 67 years and men are often affected twice as women (6). Many patients are asymptomatic at the time of diagnosis and are observed without treatment until they develop symptoms or evidence of disease progression. Lymphadenopathy occurs in approximately 80 percent and splenomegaly in approximately 50 percent of the patients at the time of diagnosis. There is a wide variation in the rate of clinical progression (7). Identification of prognostic factors by which the clinical course of the disease could be predicted, has been one of the most important research interests in B-CLL patients. Several prognostic factors have been found. One of the most reliable prognostic markers in B-CLL patients is mutational status of the immunoglobulin heavy chain variable region $(\operatorname{IgVH})$. Patients with leukemic cells, which express unmutated IgVH genes, have a greater tendency for disease progression than those whose leukemic cells express IgVH genes with less than 98 percent nucleic acid sequence homology with their germ-line counterparts $(8,9)$. However, mutational analysis of IgVH is both difficult and costly, and it is also unreachable for many clinical genetic laboratories. Therefore, finding of

Copyright (C) 2014, Iranian Red Crescent Medical Journal. This is an open-access article distributed under the terms of the Creative Commons Attribution-NonCommercial 4.0 International License (http://creativecommons.org/licenses/by-nc/4.0/) which permits copy and redistribute the material just in noncommercial usages, provided the original work is properly cited. 
some surrogate markers instead of mutational status of IgVH for the identification of disease progression has become an important issue. Cluster of differentiation (CD) markers are the first biological markers to be extensively studied as a surrogate marker; because measuring them by flow cytometry is fast and convenient. CD38 and CD23 are two markers correlating with IgVH mutational status, but their prognostic values have been a controversial issue and require further investigations (9-12). Nonetheless, high-level expression of CD38 in leukemic cells remains associated with an adverse prognosis (12, 13). Although, a research has been shown $84.2 \%$ of CD38 negative patients relate with mutated IgVH genes (14). Gene expression profiling by cDNA microarray showed that expression of several genes could be associated with IgVH mutational status in B-CLL patients. One of the most potential surrogate markers is ZAP70 (70-kDa zeta-chain associated protein kinase), a member of the Syk protein tyrosine kinase family $(14,15)$. High expression of this protein was correlated with unmutated IgVH subtype, and its low expression was observed in mutated IgVH subtype (16). However, the role of ZAP70 in pathogenesis of B-CLL is not known yet, and its correlation with IgVH mutational status is not definite. Therefore, it cannot be taken as the sole marker with high accuracy.

\section{Objectives}

In the present study, we determined ZAP70 mRNA expression level in the del17p13, del6q21 and del11q13 subgroups of Iranian B-CLL patients to investigate prognostic value of ZAP70 expression.

\section{Patients and Methods}

\subsection{Patients}

This cross sectional study was conducted in Shahrekord University of Medical Sciences, Iran (Project Number: 1389-1-70-282). The patients subjected to this survey, were enrolled using consent form between 2008 and 2010 in Imam Khomeini Hospital of Tehran. Sampling method in this study was census. A total of 71 patients were included in the study during 20 months. Five patients were excluded because of failure to grow in culture. The diagnostic inclusion criteria were based on the National Cancer Institute Working Group (NCI-WG) guidelines for diagnosis of B-CLL (17). Sex, age and Rai staging were extracted from hospital records. Two patients were diagnosed at stage I; 10; at stage II; 11 at stage III, and three at stage IV. Thirty were males and 36 cases were females. Patients were 50-70 years old. Fluorescence in situ hybridization (FISH) analysis was carried out on bone marrow or peripheral blood samples using probes for del6q21,17p13 and del11q13.

\subsection{Immunophenotyping}

By density gradient centrifugation using Ficoll-Paque, mononuclear cells were separated from heparinized venous blood. Phosphate-buffered saline (PBS) was used to wash the cells twice. Then cells were incubated with fluorescein isothiocyanate-conjugated anti-mouse antibody (IQ-Products, The Netherlands) for: CD2, CD5, CD10, CD11c, CD19, CD20, CD22, CD23, CD25, CD38, FMC7, and HLA-DR. The stained cells were sorted using the FACScan flowcytometer (Becton Dickinson, San Jose, CA, USA) and the data were analyzed using the CellQuest software.

\subsection{RNA extraction, cDNA synthesis and gene ex- pression analysis}

Two milliliters sterile PBS was used for diluting $2 \mathrm{~mL}$ EDTA-preserved fresh whole blood and carefully transferred to $4 \mathrm{~mL}$ Ficoll-Paque (Biomol, The Netherlands). The sample was centrifuged at $2000 \mathrm{rpm}$ for 15 minutes. The buffy coat was isolated in a 1.5 -mL Ependorff microtube. The specimens were normalized, and then their total RNA was extracted from the buffy coat using the High Pure RNA Isolation kit (Roche, Germany) according to manufacturer instructions. The quality and concentration of the extracted RNA samples was determined using spectrophotometer by measuring optical density at 260 and $280 \mathrm{~nm}$ (Genova Life Science Analyser-DNA, Jenway, England). In order to synthesize first-strand cDNA, random hexamer primers and a commercially available kit (Revert Aid First-Strand cDNA Synthesis kit, Fermentas, Life sciences, Germany), were used. Subsequently, cDNA was amplified by PCR with ZAP70 primers to determine the accuracy of the cDNA synthesis reaction and the PCR products were analyzed using electrophoresis on a $2 \%$ agarose gel. In order to determine ZAP70 expression, a quantitative real-time RT-PCR method was established. The amplification was performed using a real-time rotary analyzer (Rotor-Gene 6000, Corbett, Australia), and at the end of reactions data were analyzed by the RotorGene software according to the comparative $\Delta \Delta$ Ct method. A 32-year-old healthy man cDNA sample was used as a calibrator. Housekeeping gene $\beta$-glucoronidase (GUSB) was selected as the reference gene for normalization. Using 10-fold serial dilutions of the calibrator's cDNA, the researchers obtained two standard curves for ZAP70 and GUSB. Primers of ZAP70 and GUSB are shown in Table 2. Amplification of cDNA was performed by Real-time RT-PCR, in a final reaction volume of $20 \mu \mathrm{L}$ containing $2 \mu \mathrm{L}$ target cDNA, $0.7 \mu \mathrm{L}$ forward primers, $0.7 \mu \mathrm{L}$ reverse primer, $4 \mu \mathrm{L}$ master mix (LightCycler Fast Start DNA Master Sybr Green I, Roche, Germany) and $12.6 \mu \mathrm{L}$ PCR-grade water. The thermal cycling conditions were 10 minutes at $95^{\circ} \mathrm{C}$ followed by 40 cycles of denaturation at $94^{\circ} \mathrm{C}$ for 12 $\mathrm{s}$, annealing at $60^{\circ} \mathrm{C}$ for $15 \mathrm{~s}$ and extension at $72^{\circ} \mathrm{C}$ for $12 \mathrm{~s}$ for both genes. All reactions were run in duplicates.

\subsection{Statistical Analysis}

The SPSS version 19 was used to analyze raw data. To determine the statistical significance of ZAP70 expression in 
cytogenetic subgroups versus the control group, one-way ANOVA test was used. Tukey, Duncan and Scheffe were utilized as Post hoc test to categorize differences. P value less than 0.05 was considered to be statistically significant.

\section{Results}

B-type lymphocyte CD markers including CD5, CD19, CD20, CD23 and CD38 were successfully measured in all patients' peripheral blood samples using flow cytometery. Mean and range of these CD markers were collected from patients' profiles and are given in Table 1 .

ZAP70 expression from 11 patients with del17p13, 5 patients with del6q21, 10 patients with del11q13, 15 control cases and 15 undetected chromosomal abnormalities were performed using real-time PCR and cDNA samples. Size of all amplification products was of the expected size and no genomic DNA contamination was observed, this was shown by analysis of the melting curve. Performance quality of amplification was determined by standard curves. The quality of amplification performance was evaluated with the standard curves produced from 10-fold serial dilutions of the calibrator's cDNA. The efficiency value of the reactions was 0.98 to 1.05 for GUSB and 0.94 to 1.01 for ZAP70 and the correlation coefficient amplification was about one. The ratio among ZAP70 and GUSB expression automatically at the end of each reaction for each speci- men was generated by the software RotoGene. The expression ratio was measured with statistical analysis in cytogenetic subgroups and produced the subsequent results. Mean and standard deviation of the expression ratio in the control group was 1.002 and 0.320 , respectively. These two parameters in the del17p13 subgroup were 2.9 and 1.9, in the del6q21 subgroup 1.9 and 0.80 , and 2.5 and 1.0 in the del11q13.The distribution of ZAP70 expression ratio to GUSB in both cytogenetic subgroup and the control group is demonstrated in Figure 1. ANOVA displayed that there was a significant difference in the dispensation of ZAP70 expression ratio between the cytogenetic subgroups and the control group (Table 2). Expression using ANOVA test Tukey multiple comparison test shown in Table 3 indicates thatZAP70 expression in the del11q13 and del17p13are significantly difference versus control group. ZAP70 expression had about 2.88 and 2.47 fold overexpression in del17p and del11q13, respectively. But ZAP70 expression in del6q21 was the same as that of the control group (Table 3).expression using ANOVA test Tukey, multiple comparison test shown in Table 3 indicates that ZAP70 expression in the del11q13 and del17p13are significantly difference versus control group. ZAP70 expression had about 2.88 and 2.47 fold overexpression in del17p and del11q13, respectively. But ZAP70 expression in del6q21 was the same as that of the control group (Table 3 ).

Table 1. Some Hematological Characteristics of B-CLL Patients

\begin{tabular}{lcccccccccc}
\hline Subgroup & \multicolumn{2}{c}{ CD5 } & \multicolumn{2}{c}{ CD19 } & \multicolumn{2}{c}{ CD20 } & \multicolumn{2}{c}{ CD23 } & \multicolumn{2}{c}{ CD38 } \\
\hline & Mean & Range & Mean & Range & Mean & Range & Mean & Range & Mean & Range \\
\hline Del17p & $48 \pm 26.4$ & $15-92.3$ & $67 \pm 25.1$ & $22-93.0$ & $53 \pm 22.0$ & $8-79.4$ & $40 \pm 22.0$ & $1-73.8$ & $44 \pm 25.4$ & $12-84.0$ \\
Del6q & $37 \pm 19.0$ & $12-53.3$ & $57.6 \pm 12.0$ & $42-68.0$ & $32 \pm 23.2$ & $12-64.3$ & $28 \pm 34.7$ & $1-67.2$ & $23 \pm 8.2$ & $14-33.0$ \\
Del11q1 & $37 \pm 18.6$ & $4-58$ & $58 \pm 26.6$ & $16-92$ & $61 \pm 18.9$ & $32-58$ & $60 \pm 22.3$ & $0.6-82$ & $48 \pm 19.5$ & $14-74$ \\
UNdet & $47 \pm 25.0$ & $2-99.2$ & $67 \pm 22.0$ & $11-97.0$ & $27 \pm 50.5$ & $2-95.1$ & $19 \pm 54.6$ & $4-85.2$ & $31 \pm 21.5$ & $1-77.3$ \\
Total & $46 \pm 25.0$ & $2-99.2$ & $66 \pm 22.1$ & $10-97.0$ & $50 \pm 26.0$ & $2-95.1$ & $53-20.4$ & $1-85.2$ & $33 \pm 22.2$ & $1-84.6$ \\
\hline
\end{tabular}

Table 2. Statistical Analysis of ZAP70 Expression Using ANOVA Test

\begin{tabular}{|c|c|c|c|c|c|}
\hline ANOVA table & Sum of Squares & df & Mean Square & $\mathbf{F}$ & Sig. \\
\hline Treatment (between columns) & 26.18 & 4 & 6.544 & 4.48 & 0.003 \\
\hline Residual (within columns) & 74.56 & 51 & 1.462 & & \\
\hline Total & 100.7 & 55 & & & \\
\hline
\end{tabular}

Table 3. The Relation of ZAP70 Expression Gene Between 3 Cytogenetic Subgroups and Control Group Analyzed by Tukey's Multiple Comparison Test

\begin{tabular}{lcc}
\hline Cytogenetic & \multicolumn{2}{c}{ ZAP70 Gene Expression in 3 Cytogenetic Subgroup With Control } \\
\hline Subgroups & Mean difference \pm SE $^{\mathrm{a}}$ & \multicolumn{1}{c}{ P value } \\
\hline Undetected & $-0.755 \pm 0.442$ & 0.438 \\
Del6q21 & $-0.880 \pm 0.624$ & 0.625 \\
Del17p & $-1.877 \pm 0.480^{\mathrm{b}}$ & 0.002 \\
Del11q13 & $-1.466 \pm 0.494^{\mathrm{b}}$ & 0.035 \\
\hline
\end{tabular}

\footnotetext{
a The mean difference is significant at $\mathrm{P}<0.05$.
}

b Abbreviations: SE Standard error. 
Figure 1. The Histogram of ZAP70 Expression Among Different Groups Studied

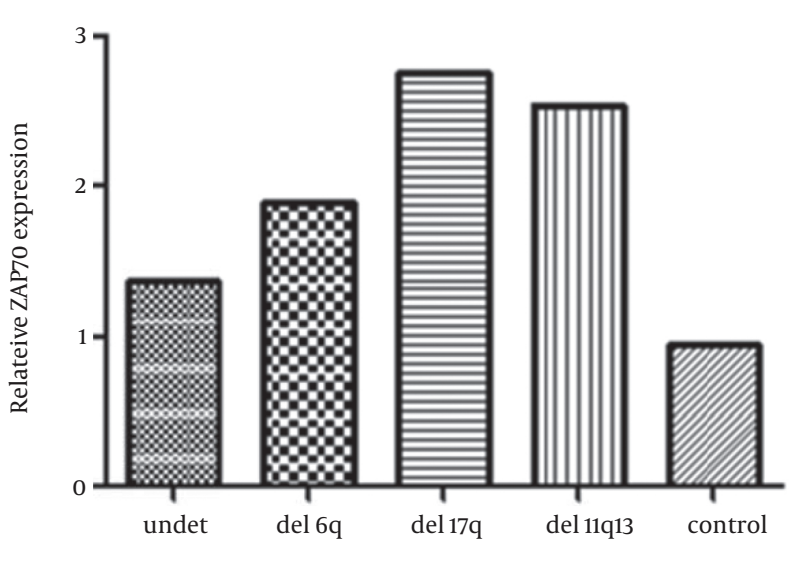

Undet $=$ undetected

\section{Discussion}

In this study, using ANOVA test, the researchers compared the ZAP70 expression between three cytogenetic subgroups and the control group. Except for the del6q21 subgroup, all of the groups consisted of at least 15 individuals. This sample number was inferred by one-way ANOVA and the sample size for each group was in this range between 15-20 individuals (18). Therefore, the sample size was considered to be satisfactory. Statistical analysis of the ZAP70 expression profile within the cytogenetic subgroups demonstrated that expression in the del17p13 and del11q13 subgroups was 2.8 and 2.4 fold more than that of control group. Comparison of means and standard deviation for the del6q21 subgroup and the control group was the same as the control group. As can be seen in Figure 1, the distribution of ZAP70 expression value within del6q21 is nearly equal compared to control and undetected subgroups with compact clustering; but this distribution within del17p13 and del11q13 has a great range compared to control and undetected groups. Studies by Teimori et al. in 2011 indicated the relationship between del13q, del11q and trisomy 12 subgroups and ZAP70 expression in CLL patients (19). In this experiment, the results of ANOVA test were significant, the findings of post hoc test showed significant differences for del17p13 and del11q13 subgroups.

Overall, this study was done on buffy coat; but Wiggers in 2014 indicated the median ZAP70 expression in NK cells was a four-fold higher and overlapped ZAP70 expression in CLL cells. Furthermore, the NK cell population was more homogeneous regarding ZAP70 expression than T cells. Therefore, they showed that ZAP70 expression in NK cells could be more valid as reference than ZAP70 expression in T cells (14). So, future studies can focus on ZAP70 NK cells to get more accurate results.

In conclusion, the relationship between these two cyto- genetic subgroups and ZAP70 expression profile was considerably significant. Thus, according to this evidence, Del 11q and del 17p could be a prognostic factor for detection of CLL patients.

\section{Acknowledgements}

Authors would like to thank all staff of molecular and cellular research center of Shahrekord who helped us in this study.

\section{Authors' Contributions}

Hamideh Jafari-Ghahfarokhi wrote the article. Saeede Ashoori and Ali Karimi performed the project. Hossein Teimori and Mohammad Taghi Akbari were the supervisors and Masoud Lotfizadeh analyzed the data.

\section{Funding/Support}

Research deputy of Shahrekord University of Medical Sciences supported this study.

\section{References}

1. Cavazzini F, Cuneo A, de Angeli C, Bardi A, Agostini P, Tammiso E, et al. Abnormalities of chromosomes 1p34-36, 4p16, 4q35, 9q11-32 and +7 represent novel recurrent cytogenetic rearrangements in chronic lymphocytic leukemia. Leuk Lymphoma. 2004;45(6):1197203.

2. Chan AC, Dalton M, Johnson R, Kong GH, Wang T, Thoma R, et al. Activation of ZAP-70 kinase activity by phosphorylation of tyrosine 493 is required for lymphocyte antigen receptor function. EMBO J.1995;14(11):2499-508.

3. Hoelzer D, Gokbuget N. T-cell lymphoblastic lymphoma and Tcell acute lymphoblastic leukemia: a separate entity? Clin Lymphoma Myeloma. 2009;9 Suppl 3:S214-21.

4. Adams RL, Cheung C, Banh R, Saal R, Cross D, Gill D, et al. Prognostic value of ZAP-70 expression in chronic lymphocytic leukemia as assessed by quantitative polymerase chain reaction and flow cytometry. Cytometry B Clin Cytom. 2014;86(2):80-90.

5. Kipps TJ. chronic lymphocytic leukemia and related diseases. ; 2010.

6. Parkin DM, Bray F, Ferlay J, Pisani P. Global cancer statistics, 2002 CA Cancer J Clin. 2005;55(2):74-108.

7. Petryk A, Peterson BA. Indolent B-cell Lymphomas. Malignant Lymphomas. 2002;94.

8. Hamblin TJ, Davis Z, Gardiner A, Oscier DG, Stevenson FK. Unmutated $\operatorname{Ig} \mathrm{V}(\mathrm{H})$ genes are associated with a more aggressive form of chronic lymphocytic leukemia. Blood.1999;94(6):1848-54

9. Claus R, Lucas DM, Stilgenbauer S, Ruppert AS, Yu L, Zucknick M et al. Quantitative DNA methylation analysis identifies a single CPG dinucleotide important for ZAP-70 expression and predictive of prognosis in chronic lymphocytic leukemia. J Clin Oncol. 2012;30(20):2483-91.

10. Damle RN, Wasil T, Fais F, Ghiotto F, Valetto A, Allen SL, et al. Ig $\mathrm{V}$ gene mutation status and CD38 expression as novel prognostic indicators in chronic lymphocytic leukemia. Blood. 1999;94(6):1840-7.

11. Hamblin TJ, Orchard JA, Ibbotson RE, Davis Z, Thomas PW, Steven son FK, et al. CD38 expression and immunoglobulin variable region mutations are independent prognostic variables in chronic lymphocytic leukemia, but CD38 expression may vary during the course of the disease. Blood. 2002;99(3):1023-9.

12. Krober A, Seiler T, Benner A, Bullinger L, Bruckle E, Lichter P, et al. $\mathrm{V}(\mathrm{H})$ mutation status, CD38 expression level, genomic aberrations, and survival in chronic lymphocytic leukemia. Blood. 2002;100(4):1410-6.

13. Schroers R, Griesinger F, Trumper L, Haase D, Kulle B, Klein-Hit- 


\section{Jafari Ghahfarokhi Het al.}

pass L, et al. Combined analysis of ZAP-70 and CD38 expression as a predictor of disease progression in B-cell chronic lymphocytic leukemia. Leukemia. 2005;19(5):750-8.

14. Wiggers TGH, Westra G, Westers TM, Abbes AP, Strunk A, KuiperKramer E, et al. ZAP70 in B-CLL cells related to the expression in NK cells is a surrogate marker for mutational status. Clinic Cytomet. 2013:n/a.

15. Crespo M, Bosch F, Villamor N, Bellosillo B, Colomer D, Rozman $\mathrm{M}$, et al. ZAP-70 expression as a surrogate for immunoglobulinvariable-region mutations in chronic lymphocytic leukemia. $N$ Engl J Med. 2003;348(18):1764-75.

16. Ferrer A, Ollila J, Tobin G, Nagy B, Thunberg U, Aalto Y, et al. Different gene expression in immunoglobulin-mutated and immuno- globulin-unmutated forms of chronic lymphocytic leukemia. Cancer Genet Cytogenet. 2004;153(1):69-72.

17. Catherwood MA, Matthews C, Niblock R, Dobbin E, Morris TC, Alexander HD. ZAP-70 mRNA quantification in B-cell chronic lymphocytic leukaemia. EurJ Haematol. 2006;76(4):294-8.

18. Cheson BD, Bennett JM, Rai KR, Grever MR, Kay NE, Schiffer CA, et al. Guidelines for clinical protocols for chronic lymphocytic leukemia: recommendations of the National Cancer Institutesponsored working group. Am J Hematol. 1988;29(3):152-63.

19. Teimori H, Akbari MT, Hamid M, Forouzandeh M, Bibordi E. Analysis of CD38 and ZAP70 mRNA expression among cytogenetic subgroups of Iranian chronic-lymphocytic-leukemia patients. Genet Mol Res. 2011;10(4):2415-23. 